\title{
Revival of Order in the Chaotic Dynamics with Position and Time Dependent Perturbed System
}

\author{
Lylyane S. Yonya Tchapda, Martin Tchoffo, Mercel Vubangsi, Faustin B. Migueu, Lukong C. Fai \\ Condensed Matter and Nanomaterials, Department of Physics, Faculty of Science, University of Dschang, Dschang, Cameroon \\ Email: lylyaneyonya@gmail.com
}

How to cite this paper: Tchapda, L.S.Y., Tchoffo, M., Vubangsi, M., Migueu, F.B. and Fai, L.C. (2020) Revival of Order in the Chaotic Dynamics with Position and Time Dependent Perturbed System. Journal of Applied Mathematics and Physics, 8, 2658-2670.

https://doi.org/10.4236/jamp.2020.811196

Received: June 9, 2020

Accepted: November 27, 2020

Published: November 30, 2020

Copyright $\odot 2020$ by author(s) and Scientific Research Publishing Inc. This work is licensed under the Creative Commons Attribution International License (CC BY 4.0).

http://creativecommons.org/licenses/by/4.0/

(c) (i) Open Access

\begin{abstract}
Studying quantum properties of a system has been quite popular in quantum mechanics. One of the most important systems that are very crucial to the framework of quantum mechanics is the system of harmonic oscillator a system whose classical evolution is known to exhibit peculiar chaotic dynamics. We are motivated to investigate the behavior of quantum properties for a system with position and time dependent perturbed. Starting with Hamiltonian, we determined the equation of motion and obtained the wave function. The energy of the whole system using the operator ordering method was found. We show that the quantum mechanical picture alludes to a chaotic dynamics as expected. This is evidenced through the appearance of energy level crossings. An additional signature to this chaotic dynamics is observed in the transition of Eigen values from real to imaginary. We also show numerically that one can give the behavior of the system is Poincare section. By so doing we confirmed that increasing and decreasing the perturbation amplitude of the system becomes chaotic.
\end{abstract}

\section{Keywords}

Schrödinger Equation, Crossing, Chaotic Dynamics, Perturbed System, Harmonic Oscillator

\section{Introduction}

The quantum harmonic oscillator $(\mathrm{QHO})$ is a problem of great interest in quantum mechanics. It is one of the very few, relatively non-trivial problems that has an exact analytic solution. The harmonic oscillator solution and algebraic formalism has applications throughout modern physics (e.g. many particle systems, 
quantum electrodynamics (QED), quantum field theory, approximations of real systems such as molecular bonds, etc.).

Harmonic oscillators are ubiquitous in physics. For example, the small vibrations of almost any mechanical system near the bottom of a potential well can usually be approximated by harmonic oscillators. This includes the case of small vibrations of a molecule about its equilibrium position or small amplitude lattice vibrations in a solid. The simple harmonic oscillator (SHO) can be used to understand a wide variety of physical phenomena ranging. However, for a real physical problem one has to introduce the perturbed harmonic oscillator. Nowadays, time and position dependent driving force present in the field of physics are most useful into the study of phenomena.

So for a long time, the study of time-dependent harmonic oscillator systems has attracted considerable attention in the literature [1] [2] [3]. The great interest in these systems is motived because they are exactly solvable quantum mechanically and can be used to successfully model many phenomena in different branches of physics, such as quantum optics [4] [5] quantum fluid dynamics [6] gravitation [7] and quantum chaos [8].

In particular, the time-dependent perturbed harmonic oscillator has been studied by various authors through different methods such as trial functions [9], path integral formulation [10] [11], Heisenberg picture approach [12], Wigner function [13], coherent and squeezed state approach [14] [15], canonical and unitary transformations [16] and invariant method combined with quadratic or linear invariant [17] [18].

The quantum-mechanical treatment of harmonic oscillator appears in the literature with [19] and without [20], a driving force. Furthermore, invariants in mechanical systems with explicitly time-dependent Hamiltonians are constants of motion of central importance in the study of dynamical systems [21]. Given a time-dependent Hamiltonian, one can obtain the corresponding time-dependent Schrödinger equation. Time dependent harmonic oscillator has several applications such as dissipative quantum tunneling effect in macroscopic system [22] and quantum motion of an ion in a Paul trap [23].

Over and above all this, in analytical study of Paul traps an ion confined within a Paul trap can be simulated, in a good approximation, with a harmonic oscillator [24]. Depending on trap parameters, the ions were found to equilibrate either as an apparently chaotic cloud or in an ordered structure.

The dynamics of a quantum system is always stable against small variations of the initial state described by the wave-function. This is due to the linearity and unitarity of the Schrödinger equation. Small variations in the Hamiltonian however can produce interesting and highly non-trivial effects on quantum time evolution such as quantum chaotic behavior. Various approaches to the quantum chaos signatures have been proposed. One notes in particular: The decay pattern of fidelity between a time evolved perturbed and unperturbed state of a system [25] [26], the correspondence between the statistics of eigenvalues and 
eigenvectors of quantum states and the canonical ensembles of the random matrix theory [27].

Though non linear Schrödinger equations have been investigated extensively within the quantum chaos domain [28] [29] [30], the chaotic dynamics of position dependent systems have received less attention. In [31], a limit cycle-chaos transition is demonstrated numerically in the forced classical PDEM duffing-type oscillator.

We seek in this work to investigate the behavior of a quantum system under the influence of external driving force with the aid of Lewis and Reisenfeld approach.This effect will be done at the level of energy, and the level of Poincare section.This paper is organized as follows. In Section 2 we present a general theory of harmonic oscillator with time and position dependent driving force, the model is presented and the solution is given. In Section 3 we analyze the chaotic effects produced in mechanical oscillators due to driving force amplitude. Finally, in Section 4 we summarize our results and conclusions.

\section{Problem Statement}

Harmonic oscillator is an important model system pervading many areas in classical physics; it is likewise ubiquitous in quantum mechanics. The non-relativistic Schrödinger equation with a harmonic oscillator potential is readily solve with standard analytic methods, whether in one or three dimensions. The harmonic oscillator at constant mass has the following parameters:

The Hamiltonian

$$
\hat{H}=\frac{\hat{p}^{2}}{2 m}+\frac{1}{2} m \omega^{2} \hat{x}^{2}
$$

The energy

$$
E_{n}=\hbar \omega\left(n+\frac{1}{2}\right)
$$

And the wave function

$$
\psi_{n}(x)=\frac{1}{\pi^{\frac{1}{4}} \sqrt{2^{n} n !}} H_{n}(x) \exp \left(-\frac{x^{2}}{2}\right)
$$

However, we will take a different tack in this work.

In quantum mechanics, perturbation theory is a set of approximation schemes directly related to mathematical perturbation for describing a complicated quantum system in terms of a simple on. The idea is to start with a simple system for which a mathematical solution is known, and add an additional perturbing Hamiltonian representing a weak disturbance to the system [32].

The quantum Hamiltonian for a time and position dependent driving force harmonic oscillator system are given by

$$
\hat{H}=\frac{\hat{p}^{2}}{2 m}+\frac{1}{2} m \omega^{2} \hat{x}^{2}+F(x, t)
$$


where $\hat{x}$ and $\hat{p}$ are the position and momentum of the mass, respectively, and $F(x, t)$ is the external driving force.

With

$$
F(x, t)=A \cos (b x+\Omega t)
$$

For which one can write the following time-dependent Schrödinger equation

$$
i \hbar \frac{\partial}{\partial t} \psi(x, t)=\left[\frac{\hat{p}^{2}}{2 m_{0}}+\frac{1}{2} m_{0} \omega^{2} \hat{x}^{2}+A \cos (b x+\Omega t)\right] \psi(x, t)
$$

Assuming the system is initially prepared in an eigenstate of the free harmonic oscillator, we include the external driving force using the mean field approximation such that the potential energy of the system can be expressed as:

$$
\begin{aligned}
V(x, t) & =\frac{1}{2} m_{0} \tilde{\omega}^{2} \hat{x}^{2}\left[1+\frac{2 A}{m_{0} \tilde{\omega}^{2}} \cos (\Omega t)\left\langle x^{-2} \sum_{j=0}^{\infty}(-1)^{j} b^{2 j} \frac{x^{2 j}}{(2 j) !}\right\rangle\right] \\
& =\frac{1}{2} m_{0} \tilde{\omega}^{2} \hat{x}^{2}[1+\beta \cos (\Omega t)]
\end{aligned}
$$

where $n$ is the quantal index the Harmonic Oscillator and

$$
\beta=\frac{2 A}{m_{0} \omega^{2}(2 n+1)} \mathrm{e}^{-\frac{b^{2}}{2 \omega}} L_{n}\left(\frac{b^{2}}{2 \omega}\right)
$$

By defining a new frequency as

$$
\tilde{\omega}^{2}=\tilde{\omega}(t)^{2}=\omega^{2}[1-\beta \cos (\Omega t)]
$$

one obtains the following effective harmonic oscillator:

$$
i \hbar \frac{\partial}{\partial t} \psi(x, t)=\left[\frac{\hat{p}^{2}}{2 m_{0}}+m_{0} \tilde{\omega}^{2} \hat{x}^{2}\right] \psi(x, t)
$$

With this manipulation, the original classical equation of motion

$$
\ddot{x}(t)+\omega^{2} x(t)=\frac{A b}{m_{0}} \sin (b x(t)+\Omega t)
$$

Now reads

$$
\ddot{x}(t)+\tilde{\omega}^{2} x(t)=0
$$

Its solution is obtained in terms of the sine elliptic $\left(S_{e}\right)$ and cosine elliptic $\left(C_{e}\right)$ functions as

$$
x(t)=K_{1} C_{e}\left[\frac{4 \omega^{2}}{\Omega^{2}},-\frac{2 \beta \omega^{2}}{\Omega^{2}}, \frac{\Omega}{2} t\right]+K_{2} S_{e}\left[\frac{4 \omega^{2}}{\Omega^{2}},-\frac{2 \beta \omega^{2}}{\Omega^{2}}, \frac{\Omega}{2} t\right]
$$

where $K_{1,2}$ are integration constants. In Figure 1 and Figure 2, we compare the analytic solution (solid curve) to the numerical solution (dotted curve) for small driving force amplitude and large driving force amplitude respectively. For small amplitude $(A)$ Figure 1, both solutions closely match while for larger amplitude $(A)$ Figure 2, the solutions show distinct behaviours.

Without loss of generality, we can set $K_{2}=i K_{1}$, then the solution can be 


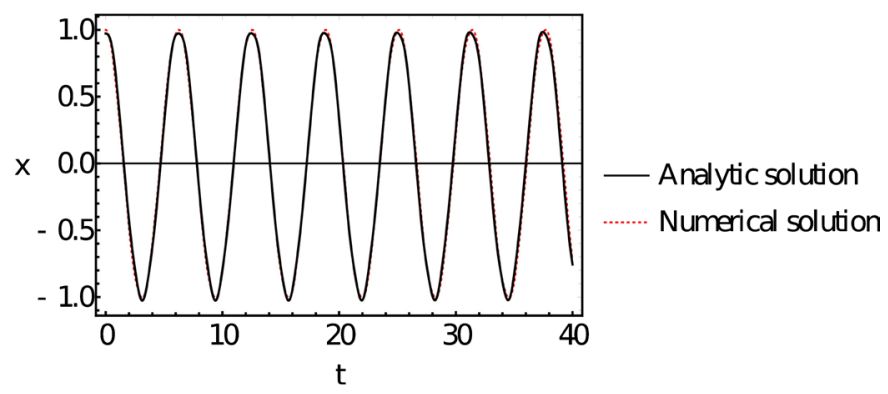

Figure 1. Comparison of the numerical solution (dotted red curve) and the analytic solution (solid curve) for large small amplitude $(A=0.5)$.

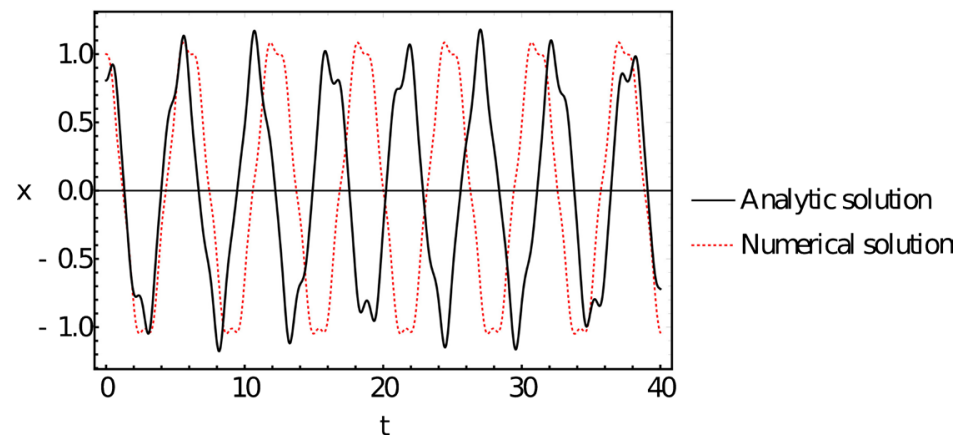

Figure 2. Comparison of the numerical solution (dotted curve) and the analytic solution (solid curve) for large perturbation amplitude $(A=10)$.

expressed in polar form as

$$
u=K_{1} r \mathrm{e}^{\mathrm{i} \theta}
$$

where $r$ and $\theta$ are time-dependent functions given by

$$
r=\left(C_{e}^{2}+S_{e}^{2}\right)^{1 / 2} ; \theta=\arctan \left(\frac{S_{e}}{C_{e}}\right)
$$

In what follows, we employ the Lewis and Reisenfeld approach to resolution of the time-dependent quantum harmonic oscillator. This consists in using the complex solution Equation (14) to construct the operators

$$
\begin{aligned}
& \hat{A}=i\left(u^{*} p-\hbar m_{0} \dot{u}^{*} x\right), \\
& \hat{A}^{\dagger}=-i\left(u p-\hbar m_{0} \dot{u} x\right),
\end{aligned}
$$

It may be easily verified that these operators satisfy the commutation relation below:

$$
\left[\hat{A}, \hat{A}^{\dagger}\right]=\hat{I}
$$

which form a pair of first order invariants that satisfy the quantum Liouville-Neumann equation

$$
\begin{aligned}
& i \frac{\partial \hat{A}}{\partial t}+[\hat{A}, H]=0 \\
& i \frac{\partial \hat{A}^{\dagger}}{\partial t}+\left[\hat{A}^{\dagger}, H\right]=0
\end{aligned}
$$


and can in turn be used to construct the Lewis quadratic invariant

$$
\hat{I}=\hat{A} \hat{A}^{\dagger}+\frac{\hbar \delta}{2}
$$

whose eigenstates $\phi_{n}^{L}$ are related to those of $H-\phi_{n}$ by

$$
\phi_{n}=\mathrm{e}^{\xi} \phi_{n}^{L}
$$

where $\xi$ are the Lewis phases. Substituting Equation (14) in Equation (12), one obtains

$$
i\left[\ddot{\theta}+2 \frac{\dot{r}}{r} \dot{\theta}\right]+\frac{\ddot{r}}{r}+\omega^{2}-\dot{\theta}^{2}=0
$$

whose imaginary part shows that $\delta=r^{2} \dot{\theta}$ is a time-invariant quantity. By requiring Equation (14) to satisfy the Wronskian condition

$$
\hbar m_{0}\left(\dot{u}^{*} u-\dot{u} u^{*}\right)=i
$$

the integral constant $K_{1}$ is obtained as

$$
K_{1}=\frac{1}{\sqrt{2 \hbar m_{0} \delta}}
$$

Once the ground state $\psi_{0}^{L}$, is obtained by solving $A \psi_{0}^{L}=0$, then the $n^{\text {th }}$ eigenstate of $\hat{I}$ follows from $\psi_{n}^{L}=\left(\frac{1}{\sqrt{n !}}\right)\left(\hat{A}^{\dagger}\right)^{n} \psi_{0}^{L}$. The $n^{\text {th }}$ eigenstate of $H$ reads:

$$
\psi_{0, n}=\left(\frac{1}{\sqrt{2^{n} n !}}\right)\left[\frac{m_{0} \delta}{\pi \hbar r^{2}}\right]^{1 / 4} H_{n}\left[\sqrt{\frac{m_{0} \delta}{\hbar r^{2}}} x\right] \exp \left[-\frac{m_{0}}{2 \hbar}\left(\frac{\delta}{r^{2}}-i \frac{\dot{r}}{r}\right) x^{2}\right] \mathrm{e}^{i \xi}
$$

The Lewis phases are given by

$$
\xi=\left\langle-\int\left[\frac{1}{\hbar} H \mathrm{~d} t\right]\right\rangle=-\int \frac{1}{\hbar} E_{n} \mathrm{~d} t
$$

The dispersion relations

$$
\begin{aligned}
& \left\langle x^{2}\right\rangle=\hbar^{2} u^{*} u=\frac{\hbar^{2} r^{2}}{2 m_{0} \delta}(2 n+1) \\
& \left\langle p^{2}\right\rangle=\hbar^{2} m_{0}^{2} \dot{u}^{*} u^{*}=\frac{\hbar m_{0}}{2 \delta}\left(\dot{r}^{2}+r^{2} \dot{\theta}^{2}\right)(2 n+1)
\end{aligned}
$$

are readily obtained, from where follows the uncertainty relation

$$
\begin{gathered}
\Delta x \Delta p=\frac{\hbar}{2}=\left[1+\frac{(\dot{r} r)}{\delta^{2}}\right]^{1 / 2}(2 n+1) \geq \frac{\hbar}{2} \\
E_{n, n}=\left(n+\frac{1}{2}\right) \frac{\hbar}{2}\left[\frac{\omega^{2}}{\dot{\theta}}+\frac{\dot{r}}{\delta} \dot{\theta}\right]
\end{gathered}
$$

\section{Results and Discussions}

Taking note of the identities 


$$
C_{e}(a, 0, x)=\cos (\sqrt{a} x) ; \quad S_{e}(a, 0, x)=\frac{1}{\sqrt{a}} \sin (\sqrt{a} x)
$$

we find that when $A=0$, the solution

$$
x(t)=K_{1} C_{e}\left[\frac{4 \omega^{2}}{\Omega^{2}}, 0, \frac{\Omega}{2} t\right]+K_{2} S_{e}\left[\frac{4 \omega^{2}}{\Omega^{2}}, 0, \frac{\Omega}{2} t\right]
$$

reduces to the solution of the classical equation of motion of the free harmonic oscillator

$$
x_{\text {fho }}(t)=K_{1} \cos (\omega t)+\frac{K_{2} \Omega}{2 \omega} \sin (\omega t)
$$

The equations in (31) for $K_{2}=\frac{2 \omega i K_{1}}{\Omega}$, therefore become

$$
r=K_{1} ; \quad \theta=\omega t
$$

substituting these quantities in Equation (28), we obtain the expected spectrum $E_{n}=\hbar \omega\left(n+\frac{1}{2}\right)$, of the free quantum harmonic oscillator.

Figure 3 shows that for increasing values of $A$ each energy level admits a critical value of the perturbation amplitude $A_{c, n}$ at which it undergoes a transition from real to complex. We found the root of the function $E_{n}(0, A, b, c)=5.01443$, in the interval $\{A, 0.172\}$ and obtained $A_{c, 0}$. For the ground state, and for the named parametrization, $A_{c, 0}=0.173$ where $E_{0}=5.01$. Beyond this value of $A_{c, 0}$, the ground state is vanishes from the spectrum.

Figure 4 shows that there are also critical values of the wavevector $b_{c, n}$ at which the eigenvalues undergo transition from complex to real.

The more excited levels however always remain real.

Small perturbation strengths influence the ground state significantly and have little effect on the more excited states as shown in Figure 5. As the perturbation strength gets very large, the ground state becomes inaccessible while the excited states are markedly altered as shown in Figure 6.

The solution $x(t)$ in the configuration:

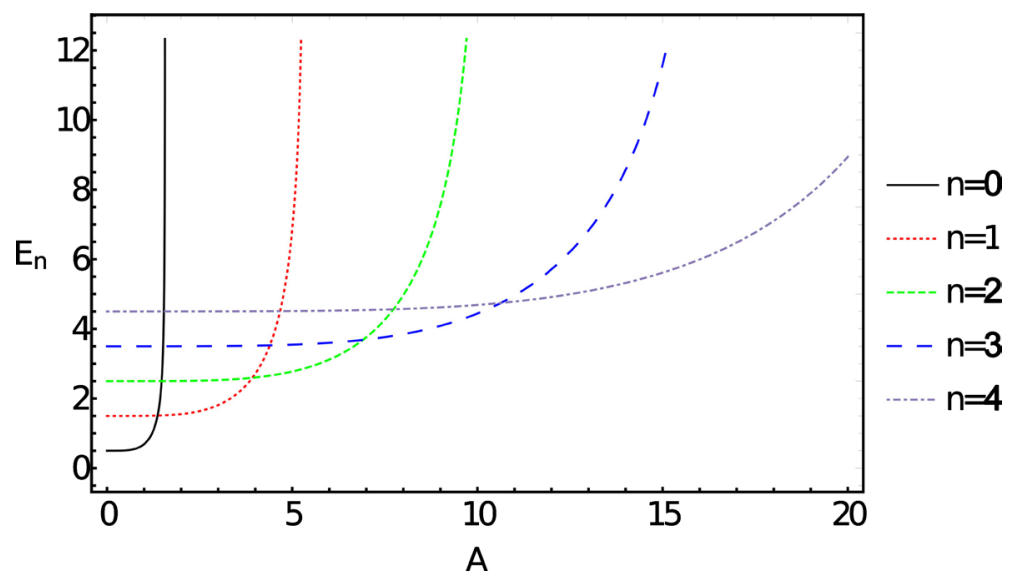

Figure 3. Plot of energy against the perturbation amplitude. 


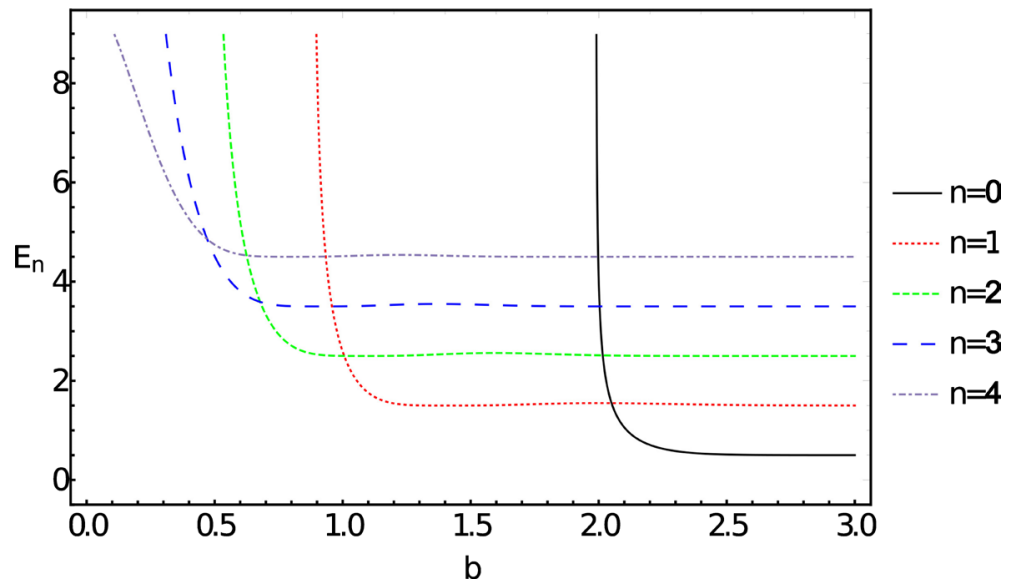

Figure 4. Plot of energy against the perturbation amplitude.

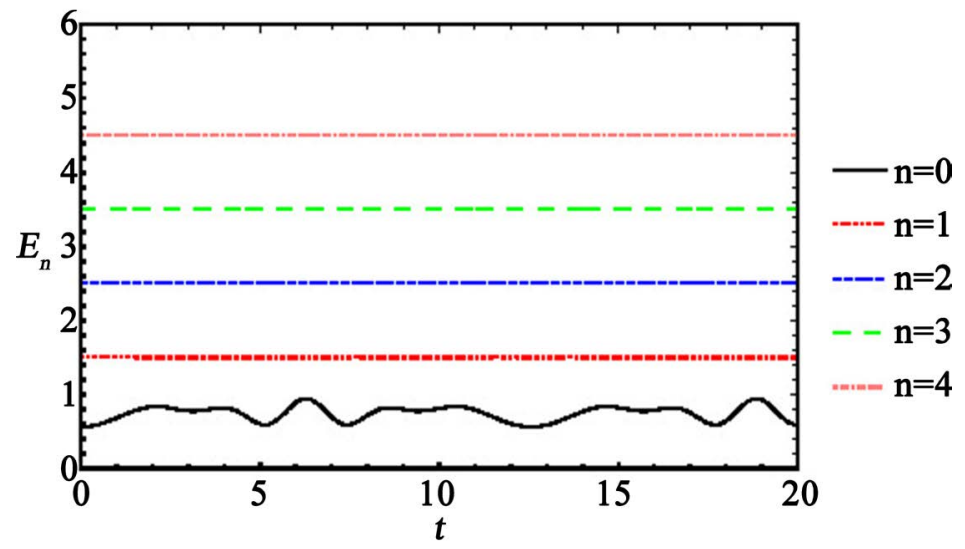

Figure 5. Plot of energy against the perturbation amplitude with small perturbation strengths.

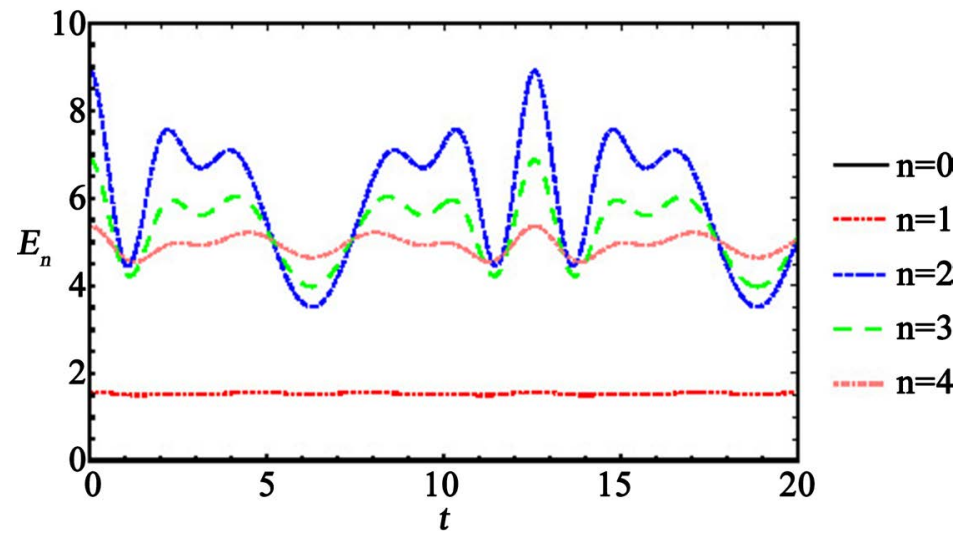

Figure 6. Plot of energy against the perturbation amplitude with large amplitude strengths.

$$
x(t)=K_{1} C_{e}\left[1,-\frac{2 \beta \omega^{2}}{\Omega^{2}}, \frac{\Omega}{2} t\right]+K_{2} S_{e}\left[1,-\frac{2 \beta \omega^{2}}{\Omega^{2}}, \frac{\Omega}{2} t\right]
$$

and

$$
x(t)=K_{1} C_{e}\left[4,-\frac{2 \beta \omega^{2}}{\Omega^{2}}, \frac{\Omega}{2} t\right]+K_{2} S_{e}\left[4,-\frac{2 \beta \omega^{2}}{\Omega^{2}}, \frac{\Omega}{2} t\right]
$$


as per the behaviour of Mathieu functions are complex valued. Equation (33) and Equation (34) translate respectively into the conditions: $\omega=\frac{\Omega}{2}$ and $\omega=\Omega$. The real and imaginary parts of $x(t)$ at these configurations oscillate with increasing amplitude which is reminiscent of parametric resonance. In these cases, the entire spectrum of the quantum mechanical problem is Imaginary.

The functions $C_{e}(a, b, z)$ and $S_{e}(a, b, z)$ are complex valued for $a>\frac{b}{2}$. This translates into the fact that we must have $\beta<1$. One is thus tempted to link the onset of an imaginary spectrum to a chaotic behavior in the classical domain.

To confirm the assertion that the system exhibits a quantum chaotic behavior, we performed a numerical experiment, the result of which shows that the system is indeed oscillatory see Figure 1 and Figure 2. An efficient pictorial tool that helps to monitor the chaotic or orderly behavior of a system is the Poincare section. The dots in such a phase space represent the state of the system at a particular instant of time. In an ordered regime, the dots get arrayed in circular tracks called KAM tori, named after Kolmogorov, Arnold and Moser who first introduced such a map.

One interesting behavior we have observed in a plane-wave perturbed harmonic oscillator with time-dependent mass is its ability to pass from an ordered regime see Figure 7(a) through chaos Figure 7(b) then back to order Figure $7(c)$ as the perturbation amplitude increases. The system however never returns to its original state.

\section{Conclusion}

It should be noted that for any of the regimes:

$$
b \gg 1 ; \Omega \gg 1 ; A \ll 1
$$

the condition $\beta<1$ is satisfied and the system approaches regular dynamics with $x(t) \approx x_{\text {fho }}(t)$. For Large amplitude $(A)$, this condition is no longer satisfied and one observes not only level crossing (which is a quantum mechanical signature of chaos) but also a transition of one eigenvalue after another from real to complex. After each transition, there is a parameter domain in which the rest of the levels above are normally ordered before the next energy level "whites out" of the spectrum. This may explain Marco Frasca's [33] observation of the system Equation (35) occasionally passing from fully developed chaos to regular motion with increasing perturbation strength. The "whiting out of levels" in the quantum mechanical system explains why in the classical counterpart, the reforming KAM tori never resemble the original. In the present observation, the appearance of complex eigen values in the quantum mechanical system is synonymous to suppression of regular dynamics in the classical system.

It has been known that on the one hand, parametric resonance can induce 

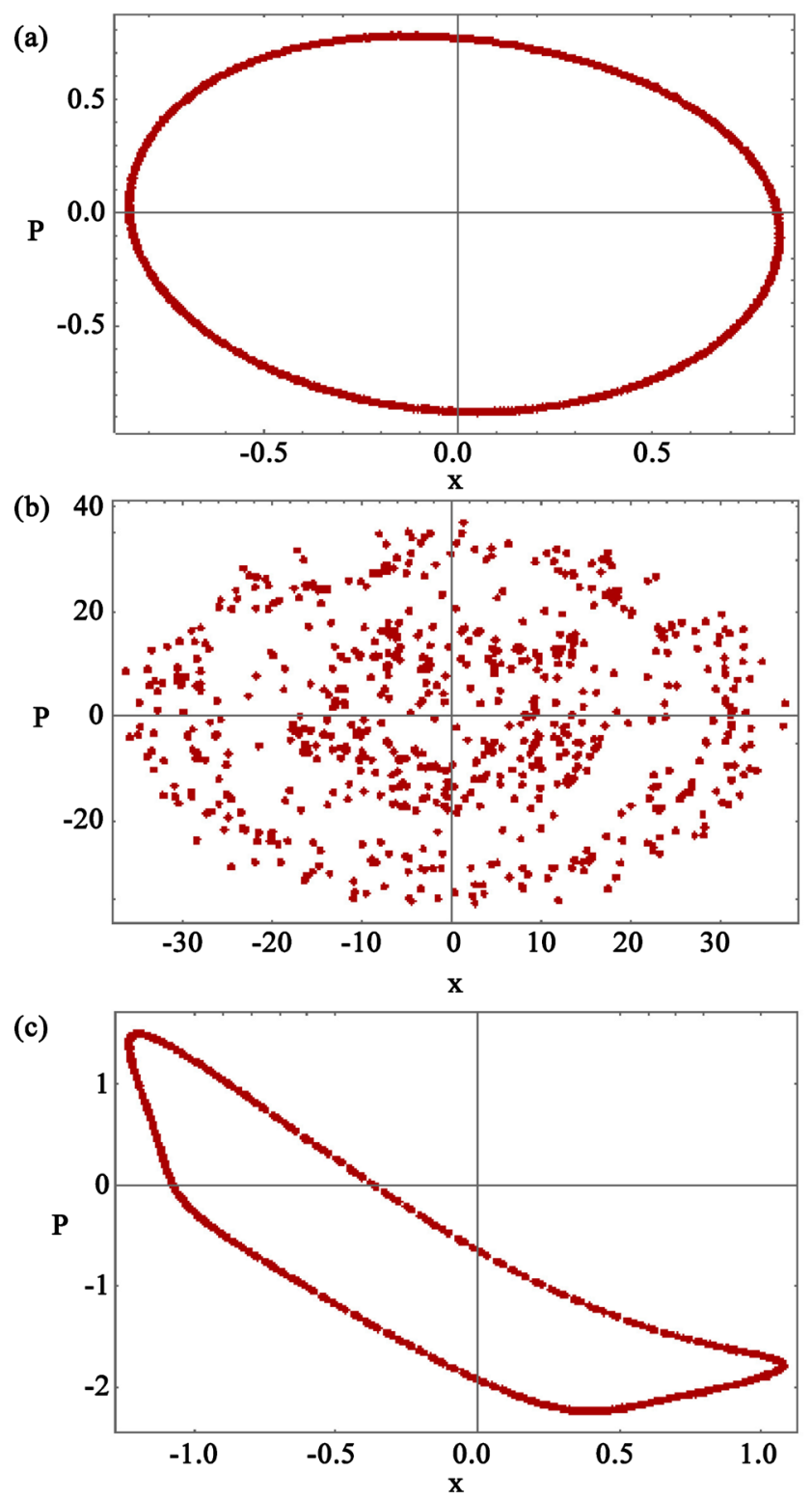

Figure 7. Poincare section for different values of amplitude $(A)$; (a) Poincare section with amplitude $(A=0.05)$; (b) Poincare section with amplitude $(A=5)$; (c) Poincare section with amplitude $(A=7)$.

chaos [34] and on the other hand it can suppress it [35] [36]. At parametric resonance, level crossing vanishes at all times for all perturbation strengths. On the other hand, whenever $\omega=\Omega / 2$ it is not possible to observe fully developed chaos in the classical system as invariant tori would always be traced in the phase space. The physical realization of such a system would have a far reaching impact in the field of Chaos control.

\section{Conflicts of Interest}

The authors declare no conflicts of interest regarding the publication of this paper. 


\section{References}

[1] Caldirola, P. (1941) Forze non conservative nella meccanica quantistica. Il Nuovo Cimento (1924-1942), 18, 393-400. https://doi.org/10.1007/BF02960144

[2] Um, C.I., Yeon, K.H. and George, T.F. (2002) The Quantum Damped Harmonic Oscillator. Physics Reports, 362, 63-192. https://doi.org/10.1016/S0370-1573(01)00077-1

[3] Gao, X.C., Xu, J.B. and Qian, T.Z. (1990) The Exact Solution for the Generalized Time-Dependent Harmonic Oscillator and Its Adiabatic Limit. Annals of Physics, 204, 235-243. https://doi.org/10.1016/0003-4916(90)90128-B

[4] Colegrave, R.K. and Abdalla, M.S. (1981) A Canonical Description of the Fabry-Pérot Cavity. Optica Acta: International Journal of Optics, 28, 495-501. https://doi.org/10.1080/713820584

[5] Pedrosa, I.A., Rosas, A. and Guedes, I. (2005) Exact Quantum Motion of a Particle Trapped by Oscillating Fields. Journal of Physics A: Mathematical and General, 38, 7757. https://doi.org/10.1088/0305-4470/38/35/009

[6] Nassar, A.B. (1984) Quantum-Fluid-Dynamics Description of the Brownian Motion. Physics Letters A, 106, 43-46. https://doi.org/10.1016/0375-9601(84)90489-4

[7] Pedrosa, I.A. and Bezerra, V.B. (1997) On the Existence of Squeezed States in an Anisotropic Universe. Modern Physics Letters A, 12, 1111-1118. https://doi.org/10.1142/S0217732397001138

[8] Loskutov, A.Yu. and Shishmarev, A.I. (1994) Control of Dynamical Systems Behavior by Parametric Perturbations: An Analytic Approach. Chaos, 4, 391. https://doi.org/10.1063/1.166017

[9] Liang, M., Yuan, B. and Zhung, K. (2002) The Exact Evolution of the Time-Dependent Driven Damped Harmonic Oscillator with Time-Dependent Mass and Frequency. Canadian Journal of Physics, 80, 1559. https://doi.org/10.1139/p02-066

[10] Jannussis, A.D., Brodimas, G.N. and Streclas, A. (1979) Propagator with Friction in Quantum Mechanics. Physics Letters A, 74, 6-10.

https://doi.org/10.1016/0375-9601(79)90566-8

[11] Song, D.Y. (2000) Periodic Hamiltonian and Berry's Phase in Harmonic Oscillators. Physical Review A, 61, Article ID: 024102. https://doi.org/10.1103/PhysRevA.61.024102

[12] Kim, H.C., Lee, M.H., Ji, J.Y. and Kim, J.K. (1996) Heisenberg-Picture Approach to the Exact Quantum Motion of a Time-Dependent Forced Harmonic Oscillator. Physical Review A, 53, 3767. https://doi.org/10.1103/PhysRevA.53.3767

[13] Akhundova, E.A. and Mukhtarov, M.A. (1995) Smoothed Wigner Function of a Quantum Damped Oscillator. Journal of Physics A: Mathematical and General, 28, 5287. https://doi.org/10.1088/0305-4470/28/18/017

[14] Lo, C.F. (1991) Generating Displaced and Squeezed Number States by a General Driven Time-Dependent Oscillator. Physical Review A, 43, 404.

https://doi.org/10.1103/PhysRevA.43.404

[15] Dodonov, V.V. and Man'ko, V.I. (1979) Coherent States and the Resonance of a Quantum Damped Oscillator. Physical Review A, 20, 550.

https://doi.org/10.1103/PhysRevA.20.550

[16] Yeon, K.H., Kim, D.H., Um, C.I., George, T.F. and Pandey, L.N. (1997) Relations of Canonical and Unitary Transformations for a General Time-Dependent Quadratic Hamiltonian System. Physical Review A, 55, 4023. 
https://doi.org/10.1103/PhysRevA.55.4023

[17] Yeon, K.H., Um, C.I. and George, T.F. (2003) Time-Dependent General Quantum Quadratic Hamiltonian System. Physical Review A, 68, Article ID: 052108.

https://doi.org/10.1103/PhysRevA.68.052108

[18] Vaidyanathan, R. (1982) Linear Invariants of a Time-Dependent Quantal Oscillator. Journal of Mathematical Physics, 23, 1346. https://doi.org/10.1063/1.525497

[19] Abdalla, S.M. (1986) Time-Dependent Harmonic Oscillator with Variable Mass under the Action of a Driving Force. Physical Review A, 34, 4598.

https://doi.org/10.1103/PhysRevA.34.4598

[20] Leach, P.G.L. (1977) Invariants and Wavefunctions for Some Time Dependent Harmonic Oscillator Type Hamiltonians. Journal of Mathematical Physics, 18, 19021907. https://doi.org/10.1063/1.523161

[21] Kaushal, R.S. and Singh, S. (2001) Construction of Complex Invariants for Classical Dynamical Systems. Annals of Physics, 288, 253-276.

https://doi.org/10.1006/aphy.2000.6108

[22] Caldeira, A.O. and Leggett, A.J. (1983) Quantum Tunneling in a Dissipative System. Annals of Physics, 149, 374-456. https://doi.org/10.1016/0003-4916(83)90202-6

[23] Brown, L.S. (1991) Quantum Motion in a Paul Trap. Physical Review Letters, 66, 527. https://doi.org/10.1103/PhysRevLett.66.527

[24] Gardiner, S.A., Cirac, J.I. and Zoller, P. (1997) Quantum Chaos in an Ion Trap: The Delta-Kicked Harmonic Oscillator. Physical Review Letters, 79, 4790. https://doi.org/10.1103/PhysRevLett.79.4790

[25] Emerson, J., Yaakov, W.S., Lloyd, S. and Cory, D.G. (2002) Fidelity Decay as an Efficient Indicator of Quantum Chaos. Physical Review Letters, 89, Article ID: 284102. https://doi.org/10.1103/PhysRevLett.89.284102

[26] Kowalewska-Kud, Å.A., Kalaga, J.K. and Leo, Å.W. (2006) Quantum Nonlinear Oscillator, Chaos and Wigner Function. Acta Physica Hungarica A: Heavy Ion Physics, 26, 243-246.

[27] Seoane, M. and Miguel Sanju, A.F. (2012) New Developments in Classical Chaotic. Reports on Progress in Physics, 76, Article ID: 016001. https://doi.org/10.1088/0034-4885/76/1/016001

[28] Larrondo, H.A. and Avalos, D.R. (1996) Dynamics of a Kicked Oscillator with a Delay in Its Parametric Feedback Loop: An Analytical Study. Nonlinear Dynamics, 11, 407-419.

[29] Mudde, R.F. and Jansz, S.G. (2003) Influence of Damping on the Delta-Kicked Harmonic Oscillator with Heaviside Kick. Physica D: Nonlinear Phenomena, 179, 1-17. https://doi.org/10.1016/S0167-2789(03)00006-X

[30] Mukhopadhyay, S., Demircioglu, B. and Chatterjee, A. (2011) Quantum Dynamics of a Nonlinear Kicked Oscillator. Nonlinear Dynamics and Systems Theory, 11, 173-182.

[31] Bagchi, B. (2013) Nonlinear Dynamics of a Position-Dependent Mass Driven Duffing-Type Oscillator. Journal of Physics A: Mathematical Theoretical, 46, Article ID: 032001. https://doi.org/10.1088/1751-8113/46/3/032001

[32] Wikipédia (2020) Perturbation Theory (Quantum Mechanics).

[33] Frasca, M. (2009) Dual Lindstedt Series and Kolmogorov-Arnol'd-Moser Theorem. Journal of Mathematical Physics, 50, Article ID: 102904.

https://doi.org/10.1063/1.3250190

[34] Khomeriki, G. (2016) Parametric Resonance Induced Chaos in Magnetic Damped 
Driven Pendulum. Physics Letters A, 380, 2382-2385.

https://doi.org/10.1016/j.physleta.2016.05.049

[35] Lima, R. and Pettini, M. (1990) Suppression of Chaos by Resonant Parametric Perturbations. Physical Review A, 41, 726. https://doi.org/10.1103/PhysRevA.41.726

[36] Fronzoni, L., Giocondo, M. and Pettini, M. (1991) Experimental Evidence of Suppression of Chaos by Resonant Parametric Perturbations. Physical Review A, 43, 6483. https://doi.org/10.1103/PhysRevA.43.6483 